Math. Model. Nat. Phenom.

Vol. 7, No. 4, 2012, pp. 64-81

DOI: $10.1051 / \mathrm{mmnp} / 20127406$

\title{
Shear-induced Electrokinetic Lift at Large Péclet Numbers
}

\author{
O. Schnitzer ${ }^{1}$, I. Frankel ${ }^{2}$, E. Yariv ${ }^{1 *}$ \\ ${ }^{1}$ Department of Mathematics, Technion - Israel \\ Institute of Technology Technion City 32000, Israel \\ ${ }^{2}$ Department of Aerospace Engineering, Technion - Israel \\ Institute of Technology Technion City 32000, Israel
}

\begin{abstract}
We analyze the problem of shear-induced electrokinetic lift on a particle freely suspended near a solid wall, subject to a homogeneous (simple) shear. To this end, we apply the large-Péclet-number generic scheme recently developed by Yariv et al. (J. Fluid Mech., Vol. 685, 2011, p. 306). For a force- and torque-free particle, the driving flow comprises three components, respectively describing (i) a particle translating parallel to the wall; (ii) a particle rotating with an angular velocity vector normal to the plane of shear; and (iiii) a stationary particle in a shear flow. Symmetry arguments reveal that the electro-viscous lift, normal to the wall, is contributed by Maxwell stresses accompanying the induced electric field, while electro-viscous drag and torque corrections, parallel to the wall, are contributed by the Newtonian stresses accompanying the induced flow. We focus upon the near-contact limit, where all electro-viscous contributions are dominated by the intense electric field in the narrow gap between the particle and the wall. This field is determined by the gap-region pressure distributions associated with the translational and rotational components of the driving Stokes flow, with the shear-component contribution directly affecting only higher-order terms. Owing to the similarity of the corresponding pressure distributions, the induced electric field for equal particle-wall zeta potentials is proportional to the sum of translation and rotation speeds. The electro-viscous loads result in induced particle velocities, normal and tangential to the wall, inversely proportional to the second power of particle-wall separation.
\end{abstract}

Keywords and phrases: electro-viscous forces, Stokes flows, singular perturbations

Mathematics Subject Classification: 76D07, 76D08

\section{Introduction}

Electrokinetic phenomena associated with the relative motion of electrolyte solutions within electric double layers adjacent to charged surfaces constitute the enabling platform in many advanced applications of micro-fluidics and nano-fluidics. An important feature of these phenomena is that the transport processes taking place within the double layer (whose characteristic scale is the nano Debye length) have global effects and their resolution is therefore essential. This universal aspect is discussed here in the context of electrokinetic lift.

*Corresponding author. E-mail: udi@technion.ac.il 
When a colloidal particle is freely suspended near a planar solid wall in the presence of simple shear, it moves parallel to the wall in the shear-flow direction, with no drift towards or away from the wall. This is a well-known consequence of the symmetry properties of Stokes flow [1,2]. Nonetheless, particle-wall repulsion was experimentally observed by Alexander \& Prieve [3]. It was speculated by Bike \& Prieve that repulsion is due to electro-viscous forces. This hypothesis was confirmed by the refined experiments of Bike et al. [4], where it was demonstrated that the lift phenomenon is appreciable in glycerol, but not in water, dismissing the possibility of an inertial mechanism.

Using a lubrication approximation, the lift problem was analyzed by Bike \& Prieve [5] for the related problem of particle translation parallel to the wall. Later, with a generic thin-Debye-layer model available [6], these authors solved the problem of shear-induced lift using bi-spherical coordinates [7]. This problem was also addressed by van de Ven and co-workers $[8,9]$.

The persistent orders-of-magnitude discrepancy between the predictions of the above models and experimental results have led Cox [10] to revisit the generic problem of streaming potential using systematic matched asymptotic expansions. Assuming that the dimensionless Debye thickness $\delta$ is small while the Hartmann and Péclet numbers are $O(1)$, Cox obtained $O\left(\delta^{6}\right)$ Maxwell stresses, while the viscous stresses associated with the flow perturbation, neglected in the generic model of Bike \& Prive [6], were found $O\left(\delta^{4}\right)$. The analysis of electrokinetic lift using Cox's scheme was carried out by Tabatabaei et al. [11].

Yariv, Schnitzer \& Frankel [12], hereafter referred to as YSF, identified an oversight in Cox [10]. Specifically, YSF showed that in the thin-Debye-layer limit the product of the Hartmann and Péclet numbers scales as $\delta^{-2}$, with the proportionality coefficient being $O(1)$. Assuming moderate Hartmann numbers, as in Cox [10], the Péclet number is accordingly $O\left(\delta^{-2}\right)$. In this limit the underlying physical mechanism giving rise to electrokinetic phenomena is the nonuniform charge convection through the Debye layer. This nonuniformity necessitates charge compensation via Ohmic currents from the electroneutral bulk, sustained by bulk electric fields (see (2.8)). The dominant convection associated with large Péclet numbers leads to a dramatic simplification of Cox's scheme. Moreover, the electro-viscous forces now turn out $O\left(\delta^{2}\right)$, and are contributed by both the electric field (through Maxwell stresses) and the velocity perturbation. The YSF scheme was recently applied to the prototype problem of particle-pair sedimentation [13].

Following the new scaling identified by YSF, and given the inconsistency of Cox's scheme, we here revisit the problem of shear-induced lift. When considering typical figures in the experiments of Alexander \& Prieve [3] it becomes evident that shear-induced lift is characterized by three disparate length-scales. Thus, the Debye thickness (in glycerol) is about $25 \mathrm{~nm}$, the sphere-wall separations are between 0.2 and $1.2 \mu \mathrm{m}$, and particle size is about $10 \mu \mathrm{m}$. These figures justify the use of the thin-Debye-layer limit, and also suggest that the near-contact limit is appropriate.

Accordingly, the goal of the present paper is to address the shear-induced lift problem using the generic scheme of YSF, with a focus on the near-contact limit. Note that our problem formulation differs from that in the lubrication analysis of Bike \& Prieve [5] in two aspects: (i) we do not specify a prescribed rectilinear velocity parallel to the wall, but rather allow it to be set by the imposed shear; and (ii) we allow for particle rotation, again with a velocity set by the shear. It turns out that the effect of rotation is comparable to that of translation.

In allowing for a force- and torque-free particle, we follow the methodology of Bike \& Prieve [7]. However, use of the YSF scheme leads to the prediction of excess drag and torque, in addition to electrokinetic lift. These electro-viscous corrections are driven by the electrokinetically induced flow, ignored by Bike $\&$ Prieve [7]. In view of the mobility relations of a sphere in proximity to a solid wall, it turns out the all three electro-viscous loads result in velocities that scale inversely with the second power of particle-wall separation.

The paper is arranged as follows. In the next section we recapitulate the generic YSF scheme and formulate the present problem. Symmetry arguments are used in $\S 3$ so as to extract some general properties of the solution. A general discussion of the problem structure in the near-contact limit is provided in $\S 4$. The case of identical particle-wall zeta potentials is analyzed in $\S 5$, where we calculate 
the lift, excess drag, and torque. The more general case of different zeta potentials is investigated in $\S 6$. We discuss the results in $\S 7$.

\section{Recapitulation and problem formulation}

\subsection{Generic scheme}

The generic paradigm of YSF considers an arbitrary zero-Reynolds-number flow of liquid electrolyte (viscosity $\mu^{*}$, permittivity $\epsilon^{*}$, equilibrium ionic concentration $c^{*}$ ) over charged solid boundaries, characterized by the externally imposed velocity scale $v^{*}$. Due to a streaming-current mechanism, the underlying Stokes flow (the 'driving' flow) results in an induced electric field which, in turn, perturbs the driving flow.

The most important parameter characterizing electrokinetic effects is the ratio

$$
\delta=\frac{1}{\kappa^{*} a^{*}}
$$

of the Debye width $1 / \kappa^{*}$, defined by

$$
\kappa^{* 2}=\frac{2 \mathcal{Z}^{2} e^{* 2} c^{*}}{\epsilon^{*} k^{*} T^{*}},
$$

and the characteristic linear dimension $a^{*}$ of the solid boundary. Here, $k^{*} T^{*}$ is the Boltzmann temperature, $e^{*}$ the elementary charge, and $\pm \mathcal{Z}$ are the (presumed equal) ionic valences.

An asymptotic analysis is carried out in the singular limit of thin double layers

$$
\delta \ll 1
$$

with the Hartmann number

$$
\lambda=\frac{2 k^{*} T^{*} a^{*} c^{*}}{\mu^{*} v^{*}}
$$

assumed $O(1)$. Using matched asymptotic expansions, the asymptotic scheme provides a macroscale description in the approximately electro-neutral 'outer' region outside the 'inner' Debye layer. This description consists of both differential equations and effective boundary conditions resulting from asymptotic matching with the Debye-scale fields. It is provided in a dimensionless notation using $a^{*}, v^{*}, \mu^{*} v^{*} / a^{*}$ and the thermal voltage $\varphi^{*}=k^{*} T^{*} / \mathcal{Z} e^{*}$ as units of length, velocity, stress and electric potential, respectively, whereby forces and torques are respectively normalized by $\mu^{*} v^{*} a^{*}$ and $\mu^{*} v^{*} a^{* 2}$.

Once the velocity $\mathbf{v}$, pressure $p$ and potential $\varphi$ are evaluated, the resulting forces may be calculated using the combination of the Newtonian

$$
\mathrm{N}=-p \mathbf{l}+(\boldsymbol{\nabla} \mathbf{v})+(\boldsymbol{\nabla} \mathbf{v})^{\dagger}
$$

and Maxwell

$$
\mathrm{M}=\lambda \delta^{2}\left(\nabla \varphi \nabla \varphi-\frac{1}{2} \nabla \varphi \cdot \nabla \varphi \mathrm{I}\right)
$$

stress tensors. In these expressions I denotes the dyadic idemfactor and the superscript $\dagger$ indicates transposition.

The pertinent macroscale fields are expanded in the generic form

$$
f \sim f_{0}+\delta^{2} f_{1}+\cdots .
$$

The driving flow $\left(\mathbf{v}_{0}, p_{0}\right)$ is governed by the Stokes equations and a no-slip condition on the charged boundaries. The induced potential $\varphi_{0}$ is governed by Laplace's equation and the inhomogeneous Neumann condition

$$
\frac{\partial \varphi_{0}}{\partial n}=\frac{\alpha \zeta}{\lambda} \frac{\partial p_{0}}{\partial n}
$$




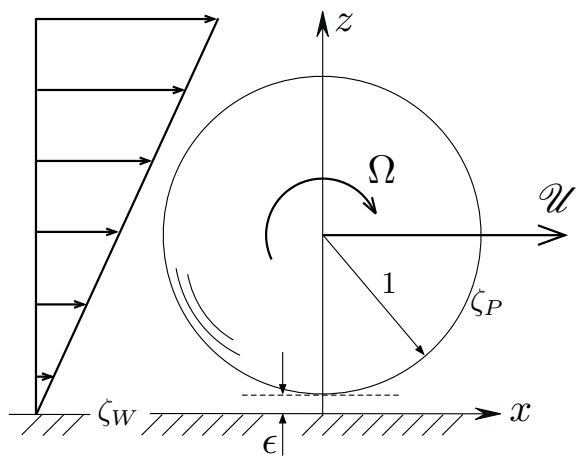

FiguRE 1. Schematic.

representing the balance between nonuniform charge convection and Ohmic double-layer charging. The induced flow $\left(\mathbf{v}_{1}, p_{1}\right)$ is also governed by the Stokes equations. Unlike the driving flow, it satisfies a Smoluchowski-type slip condition

$$
\mathbf{v}_{1}=\lambda \zeta \nabla_{s} \varphi_{0}
$$

( $\nabla_{s}$ being the surface-gradient operator) on the charged boundaries.

\subsection{Problem formulation}

The problem considered in this paper entails the motion of a spherical particle (radius $a^{*}$ ) suspended in an electrolyte solution near a planar wall. Both the particle and the wall are dielectric solids possessing uniform charge distributions. The electrolyte undergoes simple shear flow characterized by the shear rate $G^{*}$. At the instant we consider, the particle-wall separation is $\epsilon a^{*}$. The problem is conveniently handled using the Cartesian coordinates $\left(a^{*} x, a^{*} y, a^{*} z\right)$, with $z=0$ coinciding with the wall, the $z$-axis passing through the particle center, and the $x$-axis pointing in the direction of unperturbed shear flow. We also employ cylindrical coordinates $\left(a^{*} r, \theta, a^{*} z\right)$. The particle-wall configuration and the various coordinates are described in Figure 1.

Using our dimensionless scheme with $v^{*}=a^{*} G^{*}$, the driving flow $\left(\mathbf{v}_{0}, p_{0}\right)$ satisfies the continuity and Stokes equations,

$$
\nabla \cdot \mathbf{v}_{0}=0, \quad \nabla^{2} \mathbf{v}_{0}=\nabla p_{0}
$$

the no-slip condition on the wall,

$$
\mathbf{v}_{0}=\mathbf{0} \quad \text { at } \quad z=0,
$$

and the far-field condition

$$
\mathbf{v}_{0} \sim \mathrm{G} \cdot \mathbf{x} \text { as }|\mathbf{x}| \rightarrow \infty,
$$

where $\mathbf{x}=(x, y, z)$ is the position vector and

$$
\mathrm{G}=\hat{\mathbf{e}}_{x} \hat{\mathbf{e}}_{z} .
$$

Furthermore, the no-slip condition on the particle surface implies that $\mathbf{v}_{0}$ corresponds there to a rigidbody motion (see (3.1)), whose translational and rotational velocities are obtained from the condition that particle is force- and torque-free

$$
\mathbf{F}_{0}=\frac{1}{6 \pi} \oint_{\mathscr{P}} d A \hat{\mathbf{n}} \cdot \mathrm{N}_{0}=\mathbf{0}, \quad \mathbf{T}_{0}=\frac{1}{8 \pi} \oint_{\mathscr{P}} d A \hat{\mathbf{n}} \times\left(\hat{\mathbf{n}} \cdot \mathrm{N}_{0}\right)=\mathbf{0},
$$

where $\mathscr{P}$ is the particle boundary (a unit sphere) and

$$
\mathrm{N}_{0}=-p_{0} \mathbf{I}+\left(\boldsymbol{\nabla} \mathbf{v}_{0}\right)+\left(\boldsymbol{\nabla} \mathbf{v}_{0}\right)^{\dagger}
$$


is the Newtonian stress associated with the driving flow (cf. (2.5)).

The induced electric potential $\varphi_{0}$ satisfies Laplace's equation

$$
\nabla^{2} \varphi_{0}=0
$$

the Neuamnn condition (2.8) on the particle and wall, and a decay condition at large $|\mathbf{x}|$. The induced flow $\left(\mathbf{v}_{1}, p_{1}\right)$ satisfies the continuity and Stokes equations

$$
\nabla \cdot \mathbf{v}_{1}=0, \quad \nabla^{2} \mathbf{v}_{1}=\nabla p_{1}
$$

the slip condition (2.9) on the particle and wall, and a decay condition at large $|\mathbf{x}|$.

Our interest lies in the leading electro-viscous force and torque (see also $§ 7$ )

$$
\mathbf{F}_{1}=\frac{1}{6 \pi} \oint_{\mathscr{P}} d A \hat{\mathbf{n}} \cdot\left(\mathrm{M}_{1}+\mathrm{N}_{1}\right), \quad \mathbf{T}_{1}=\frac{1}{8 \pi} \oint_{\mathscr{P}} d A \hat{\mathbf{n}} \times\left[\hat{\mathbf{n}} \cdot\left(\mathrm{M}_{1}+\mathrm{N}_{1}\right)\right]
$$

wherein

$$
\mathrm{M}_{1}=\lambda\left(\nabla \varphi_{0} \nabla \varphi_{0}-\frac{1}{2} \nabla \varphi_{0} \cdot \nabla \varphi_{0} \mathrm{I}\right)
$$

is the induced Maxwell stress and

$$
\mathrm{N}_{1}=-p_{1} \mathrm{I}+\left(\boldsymbol{\nabla} \mathbf{v}_{1}\right)+\left(\boldsymbol{\nabla} \mathbf{v}_{1}\right)^{\dagger}
$$

is the induced Newtonian stress.

Note that for identical zeta potentials on the particle and the wall $\varphi_{0}$ need not be explicitly calculated since $(2.8)$ yields $[7]$

$$
\varphi_{0}=\frac{\alpha \zeta}{\lambda} p_{0}
$$

whereby (2.9) becomes

$$
\mathbf{v}_{1}=\alpha \zeta^{2} \nabla_{s} p_{0}
$$

\section{Symmetry arguments}

\subsection{Driving flow}

Since the driving flow problem is linear and homogeneous in the imposed shear $\mathrm{G}$ the particle velocities must be linear in G. The appropriate expressions may thus only be formed via products of first- and third-order geometry-dependent tensors with G. Since the problem geometry introduces a single constant vector, $\hat{\mathbf{e}}_{z}$, the only true third-order tensors one can form are l| $\hat{\mathbf{e}}_{z}$ (and permutations thereof) and $\hat{\mathbf{e}}_{z} \hat{\mathbf{e}}_{z} \hat{\mathbf{e}}_{z}$. It follows that the particle center can only translate in the $x$-direction, say with linear velocity $\mathscr{U}$. Similarly, the only geometry-dependent third-order pseudo-tensors are the isotropic triadic $\boldsymbol{\epsilon}$ and $\boldsymbol{\epsilon} \cdot \hat{\mathbf{e}}_{z} \hat{\mathbf{e}}_{z}$ (and permutations thereof), implying that the particle can only rotate in the $y$-direction, say with angular velocity $\Omega$.

It follows that the no-slip condition on the particle boundary is

$$
\mathbf{v}_{0}=\hat{\mathbf{e}}_{x} \mathscr{U}+\Omega \hat{\mathbf{e}}_{y} \times \hat{\mathbf{n}} \quad \text { on } \mathscr{P} .
$$

This condition, together with (2.12), suggests the decomposition of the driving flow into three subfields,

$$
\mathbf{v}_{0}=\mathbf{v}_{0, S}+\mathscr{U} \mathbf{v}_{0, T}+\Omega \mathbf{v}_{0, R}, \quad p_{0}=p_{0, S}+\mathscr{U} p_{0, T}+\Omega p_{0, R},
$$


with $\left(\mathbf{v}_{0, S}, p_{0, S}\right)$ corresponding to a stationary particle in a shear flow, while $\left(\mathbf{v}_{0, T}, p_{0, T}\right)$ and $\left(\mathbf{v}_{0, R}, p_{0, R}\right)$ respectively correspond to translation in the $x$-direction and rotation about the $y$-direction (in the absence of shear). Equation (2.15) then provides a similar decomposition for $\mathrm{N}_{0}$, implying in turn the decompositions of both $\mathbf{F}_{0}$ and $\mathbf{T}_{0}$ (see (2.14)); by the preceding symmetry arguments, all sub-forces are in the $x$-direction,

$$
\mathbf{F}_{0, S}=\hat{\mathbf{e}}_{x} F_{0, S}, \quad \mathbf{F}_{0, T}=\hat{\mathbf{e}}_{x} F_{0, T}, \quad \mathbf{F}_{0, R}=\hat{\mathbf{e}}_{x} F_{0, R},
$$

while all sub-torques are in the $y$-direction,

$$
\mathbf{T}_{0, S}=\hat{\mathbf{e}}_{y} T_{0, S}, \quad \mathbf{T}_{0, T}=\hat{\mathbf{e}}_{y} T_{0, T}, \quad \mathbf{T}_{0, R}=\hat{\mathbf{e}}_{y} T_{0, R}
$$

All three subfields satisfy the homogenous Stokes equations (2.10) and no-slip condition (2.11) on the wall. On the particle they satisfy (cf. (3.1))

$$
\mathbf{v}_{0, S}=\mathbf{0}, \quad \mathbf{v}_{0, T}=\hat{\mathbf{e}}_{x}, \quad \mathbf{v}_{0, R}=\hat{\mathbf{e}}_{y} \times \hat{\mathbf{n}},
$$

while at large distances (cf. (2.12))

$$
\mathbf{v}_{0, S} \sim \hat{\mathbf{e}}_{x} z, \quad \mathbf{v}_{0, T} \rightarrow \mathbf{0}, \quad \mathbf{v}_{0, R} \rightarrow \mathbf{0} .
$$

The force- and torque-free conditions (2.14) now read

$$
F_{0, S}+\mathscr{U} F_{0, T}+\Omega F_{0, R}=0, \quad T_{0, S}+\mathscr{U} T_{0, T}+\Omega T_{0, R}=0 .
$$

The symmetry of the driving flow admits the description in cylindrical coordinates of the pressure and $(r, \theta, z)$ velocity components

$$
\bar{p}_{0} \cos \theta, \quad\left\{\bar{u}_{0} \cos \theta, \bar{v}_{0} \sin \theta, \bar{w}_{0} \cos \theta\right\},
$$

wherein $\bar{p}_{0}, \bar{u}_{0}, \bar{v}_{0}$ and $\bar{w}_{0}$ are functions of $z$ and $r$ (and $\epsilon$ ), independent of $\theta$. This description separately applies to each of the subfields in the decomposition (3.2). Thus, for instance, the pressure and $(r, \theta, z)$ velocity components of the shear subfield $\left(p_{0, S}, \mathbf{v}_{0, S}\right)$ adopt the form

$$
\bar{p}_{0, S} \cos \theta, \quad\left\{\bar{u}_{0, S} \cos \theta, \bar{v}_{0, S} \sin \theta, \bar{w}_{0, S} \cos \theta\right\}
$$

where $\bar{p}_{0, S}, \bar{u}_{0, S}, \bar{v}_{0, S}$ and $\bar{w}_{0, S}$ are functions of $z$ and $r$.

The continuity and Stokes equations (2.10) governing $\left(\mathbf{v}_{0}, p_{0}\right)$ now appear

$$
\frac{\partial \bar{u}_{0}}{\partial r}+\frac{\bar{u}_{0}+\bar{v}_{0}}{r}+\frac{\partial \bar{w}_{0}}{\partial z}=0
$$

and

$$
\begin{gathered}
\frac{\partial \bar{p}_{0}}{\partial r}=\frac{\partial^{2} \bar{u}_{0}}{\partial r^{2}}+\frac{1}{r} \frac{\partial \bar{u}_{0}}{\partial r}-2 \frac{\bar{u}_{0}+\bar{v}_{0}}{r^{2}}+\frac{\partial^{2} \bar{u}_{0}}{\partial z^{2}}, \\
-\frac{\bar{p}_{0}}{r}=\frac{\partial^{2} \bar{v}_{0}}{\partial r^{2}}+\frac{1}{r} \frac{\partial \bar{v}_{0}}{\partial r}-2 \frac{\bar{u}_{0}+\bar{v}_{0}}{r^{2}}+\frac{\partial^{2} \bar{v}_{0}}{\partial z^{2}}, \\
\frac{\partial \bar{p}_{0}}{\partial z}=\frac{\partial^{2} \bar{w}_{0}}{\partial r^{2}}+\frac{1}{r} \frac{\partial \bar{w}_{0}}{\partial r}-\frac{\bar{w}_{0}}{r^{2}}+\frac{\partial^{2} \bar{w}_{0}}{\partial z^{2}},
\end{gathered}
$$

respectively. Similar equations govern each of the three subfields. 


\subsection{Induced fields}

Consider now the induced fields. The problem governing $\varphi_{0}$ is linear and homogeneous in the driving flow (and hence in G) through the Neumann condition (2.8). The slip condition (2.9) then implies that the same linear dependency also holds for the induced flow. Two consequences readily follow: (i) The quadratic Maxwell stress (2.19) is linear in the fourth-order tensor GG: considering all possible geometrical third- and fifth-order true tensors, this linear dependence can only result in a force in the $z$-direction (lift) and no torque; (ii) With the induced Newtonian stress being linear in G, the symmetry relations governing the driving flow apply also to the induced one: the force and torque contributions thus act in the $x$ - and $y$-directions, respectively.

We therefore decompose the electro-viscous force

$$
\mathbf{F}_{1}=\hat{\mathbf{e}}_{z} L_{1}-\hat{\mathbf{e}}_{x} D_{1}
$$

with the lift contributed by the induced Maxwell stress

$$
L_{1}=\frac{1}{6 \pi} \hat{\mathbf{e}}_{z} \cdot \oint_{\mathscr{P}} d A \hat{\mathbf{n}} \cdot \mathrm{M}_{1}
$$

and the excess drag contributed by the induced Newtonian stress

$$
D_{1}=-\frac{1}{6 \pi} \hat{\mathbf{e}}_{x} \cdot \oint_{\mathscr{P}} d A \hat{\mathbf{n}} \cdot \mathrm{N}_{1} .
$$

Similarly, the electro-viscous torque

$$
\mathbf{T}_{1}=\hat{\mathbf{e}}_{y} T_{1}
$$

is contributed by the induced Newtonian stress

$$
T_{1}=\frac{1}{8 \pi} \hat{\mathbf{e}}_{y} \cdot \oint_{\mathscr{P}} d A \hat{\mathbf{n}} \times\left(\hat{\mathbf{n}} \cdot \mathrm{N}_{1}\right) .
$$

Useful cylindrical-coordinate representations also apply for the induced fields. Thus, the linear boundary condition (2.8) and the representation (3.8) implies that

$$
\varphi_{0}(z, r, \theta ; \epsilon)=\bar{\varphi}_{0}(z, r ; \epsilon) \cos \theta
$$

whereby Laplace's equation (2.16) becomes

$$
\frac{\partial^{2} \bar{\varphi}_{0}}{\partial r^{2}}+\frac{1}{r} \frac{\partial \bar{\varphi}_{0}}{\partial r}-\frac{\bar{\varphi}_{0}}{r^{2}}+\frac{\partial^{2} \bar{\varphi}_{0}}{\partial z^{2}}=0
$$

Similarly, the linear slip condition (2.9) implies that the induced pressure $p_{1}$ and velocity $\mathbf{v}_{1}$ possess the representations (cf. (3.8)),

$$
\bar{p}_{1} \cos \theta, \quad\left\{\bar{u}_{1} \cos \theta, \bar{v}_{1} \sin \theta, \bar{w}_{1} \cos \theta\right\}
$$

where $\bar{p}_{1}, \bar{u}_{1}, \bar{v}_{1}$ and $\bar{w}_{1}$ are functions of $z$ and $r$. Since the induced flow satisfies the same differential equations (2.17) as the driving flow, the fields $\bar{p}_{1}, \bar{u}_{1}, \bar{v}_{1}$ and $\bar{w}_{1}$ are governed by equations similar to (3.11):

$$
\begin{gathered}
\frac{\partial \bar{u}_{1}}{\partial r}+\frac{\bar{u}_{1}+\bar{v}_{1}}{r}+\frac{\partial \bar{w}_{1}}{\partial z}=0 \\
\frac{\partial \bar{p}_{1}}{\partial r}=\frac{\partial^{2} \bar{u}_{1}}{\partial r^{2}}+\frac{1}{r} \frac{\partial \bar{u}_{1}}{\partial r}-2 \frac{\bar{u}_{1}+\bar{v}_{1}}{r^{2}}+\frac{\partial^{2} \bar{u}_{1}}{\partial z^{2}}, \\
-\frac{\bar{p}_{1}}{r}=\frac{\partial^{2} \bar{v}_{1}}{\partial r^{2}}+\frac{1}{r} \frac{\partial \bar{v}_{1}}{\partial r}-2 \frac{\bar{u}_{1}+\bar{v}_{1}}{r^{2}}+\frac{\partial^{2} \bar{v}_{1}}{\partial z^{2}}, \\
\frac{\partial \bar{p}_{1}}{\partial z}=\frac{\partial^{2} \bar{w}_{1}}{\partial r^{2}}+\frac{1}{r} \frac{\partial \bar{w}_{1}}{\partial r}-\frac{\bar{w}_{1}}{r^{2}}+\frac{\partial^{2} \bar{w}_{1}}{\partial z^{2}} .
\end{gathered}
$$




\section{Near-contact configurations: Preliminaries}

When the gap between the particle and the wall is narrow,

$$
\epsilon \ll 1,
$$

it is natural to analyze the problem using inner-outer asymptotic expansions. The inner gap region is where

$$
z=O(\epsilon), \quad r=O\left(\epsilon^{1 / 2}\right),
$$

and is naturally described by the strained coordinates [14]

$$
z=\epsilon Z, \quad r=\epsilon^{1 / 2} R
$$

In these coordinates, the particle surface is provided by

$$
Z=H(R)
$$

wherein $H(R)=H_{0}(R)+O(\epsilon)$, with

$$
H_{0}=1+\frac{R^{2}}{2} .
$$

In the outer region the sphere appears at leading order to be in contact with the wall.

The translation and rotation subfields appearing in (3.2) were already calculated in that fashion by O'Neill \& Stewartson [15] and Cooley \& O'Neill [16], respectively. In both problems the relative motion is associated with intense $O\left(\epsilon^{-1}\right)$ velocity gradients in the gap which, through a lubrication mechanism, lead to an $O\left(\epsilon^{-3 / 2}\right)$ pressure there. This pressure results in $O(\ln \epsilon)$ drag force [17]; the subsequent $O(1)$ terms are contributed by both the inner and outer domains. Considering logarithmic terms as being $O(1)$ [18] the leading-order forces are contributed by both asymptotic regions.

In the induced electro-viscous fields, the gap singularity is expected to be more dramatic. In view of the Neumann condition (2.8), the induced electric field is expected to be $O\left(\epsilon^{-2}\right)$, whereby the slip condition (2.9) leads to induced flow of the same magnitude; by a lubrication approximation the pressure perturbation turns out to scale as $\epsilon^{-7 / 2}$. Furthermore, due to the quadratic dependence of Maxwell stress on electric field, we anticipate it to scale as $\epsilon^{-4}$. With the gap-region area scaling as $\epsilon$, these intense stresses suggest that the electro-viscous forces are dominated by the inner gap contribution.

Remarkably, the shear subfield, which is the one animating the driving flow, does not contribute to this singular behavior. Indeed, since the problem of a stationary particle under shear is not directly associated with intense velocity gradients in the gap, its analysis [19,20] does not require the separation to inner and outer regions, as the 'outer' fields do not become singular near the 'contact point.' Indeed, inspection of the resulting fields in [20] as they approach the 'contact point' reveals that the corresponding pressure is regular there.

We therefore need only consider the translation and rotation subfields. In what follows, we next present some of the key elements in the analyses of O'Neill \& Stewartson [15] and Cooley \& O'Neill [16].

\subsection{Driving flow}

In the translation problem the velocity components parallel to wall are $O(1)$ in the gap region (4.2); the continuity equation (3.10) in conjunction with wall impermeability then implies an $O\left(\epsilon^{1 / 2}\right)$ axial velocity component. These estimates suggest the definition of the rescaled variables

$$
\bar{u}_{0, T}=U_{0, T}(Z, R ; \epsilon), \quad \bar{v}_{0, T}=V_{0, T}(Z, R ; \epsilon), \quad \bar{w}_{0, T}=\epsilon^{1 / 2} W_{0, T}(Z, R ; \epsilon) ;
$$

furthermore, momentum balance parallel to the wall implies $O\left(\epsilon^{-3 / 2}\right)$ large pressure, suggesting the rescaling

$$
\bar{p}_{0, T}=\epsilon^{-3 / 2} P_{0, T}(Z, R ; \epsilon) .
$$


Substituting these expansions together with (4.3) into the continuity and Stokes equations (3.10)-(3.11) yield

$$
\frac{\partial U_{0, T}}{\partial R}+\frac{U_{0, T}+V_{0, T}}{R}+\frac{\partial W_{0, T}}{\partial Z}=0
$$

together with

$$
\frac{\partial P_{0, T}}{\partial R}=\frac{\partial^{2} U_{0, T}}{\partial Z^{2}}+O(\epsilon), \quad-\frac{P_{0, T}}{R}=\frac{\partial^{2} V_{0, T}}{\partial Z^{2}}+O(\epsilon), \quad \frac{\partial P_{0, T}}{\partial Z}=\epsilon \frac{\partial^{2} W_{0, T}}{\partial Z^{2}}+O\left(\epsilon^{2}\right) .
$$

The boundary conditions are

$$
U_{0, T}=V_{0, T}=W_{0, T}=0
$$

at $Z=0$ and

$$
U_{0, T}=1, \quad V_{0, T}=-1, \quad W_{0, T}=0
$$

at $Z=H$.

Each of the functions $U_{0, T}, V_{0, T}, W_{0, T}$, and $P_{0, T}$ possess the generic expansion

$$
F(Z, R ; \epsilon) \sim F^{(0)}(Z, R)+\epsilon F^{(1)}(Z, R)+\cdots .
$$

The leading-order balance of (4.7b) implies that $P_{0, T}^{(0)}$ is independent of $Z$,

$$
P_{0, T}^{(0)}=P^{*}(R) .
$$

The function $P^{*}$ was calculated by O'Neill \& Stewartson [15] using the remaining leading-order equations

$$
P^{*}=\frac{6 R}{5 H_{0}^{2}}
$$

The corresponding leading-order axial velocity is

$$
W_{0, T}^{(0)}=\frac{8 R-2 R^{3}}{5 H_{0}^{4}} Z^{3}+\frac{2 R^{3}-8 R}{5 H_{0}^{3}} Z^{2} .
$$

(There is a minor typographical error in the corresponding expression provided by O'Neill \& Stewartson [15].)

The scaling of the rotation-subfield variables is the same as (4.6), namely

$$
\begin{aligned}
& \bar{u}_{0, R}=U_{0, R}(Z, R ; \epsilon), \bar{v}_{0, R}=V_{0, R}(Z, R ; \epsilon), \quad \bar{w}_{0, R}=\epsilon^{1 / 2} W_{0, R}(Z, R ; \epsilon), \\
& \bar{p}_{0, R}=\epsilon^{-3 / 2} P_{0, R}(Z, R ; \epsilon) .
\end{aligned}
$$

The functions $U_{0, R}, V_{0, R}, W_{0, R}$ and $P_{0, R}$ satisfy the same differential equations (4.7), and wall conditions (4.8) as their translational counterparts; because of rotation, the particle-surface condition (4.9) is now replaced by

$$
U_{0, T}=-1, \quad V_{0, T}=1, \quad W_{0, T}=-R .
$$

As in the the translation problem, the leading-order pressure $P_{0, R}^{(0)}$ is independent of $Z$. As a matter of fact, its evaluation yields [16]

$$
P_{0, R}^{(0)}=P^{*}(R)
$$

with $P^{*}$ given by (4.12); the corresponding axial velocity is however different,

$$
W_{0, R}^{(0)}=\frac{8 R-2 R^{3}}{5 H_{0}^{4}} Z^{3}-\frac{26 R+R^{3}}{10 H_{0}^{3}} Z^{2} .
$$


Intuitively, it may appear that a unit linear velocity in the $x$-direction and a unit angular velocity in the $y$-direction should result in mutually canceling pressure distributions, since the relative tangential velocities between the particle surface and the wall are in opposing directions in the gap (see Figure 1). Note however that the rotation problem also introduces a non-zero axial velocity in the boundary condition (4.16) with the resulting distributions of radial and tangential velocities being different. Inspection of the analyses of O'Neill \& Stewartson [15] and Cooley \& O'Neill [16] actually suggests that the identical pressure in both problems is fortuitous.

In the translation and rotation subfields, the leading-order inner-region contribution to the force and torque is $O(\ln \epsilon)$. The subsequent $O(1)$ terms are contributed by both the inner and outer regions. In the shear subfields, the leading $O(1)$ force and torque are obviously contributed by the outer region. Summarizing the findings of O'Neill \& Stewartson [15] and Cooley \& O'Neill [16] yields the forces

$$
F_{0, S} \approx 1.7009, \quad F_{0, T} \approx-\frac{8}{15} \ln \frac{2}{\epsilon}-0.58461, \quad F_{0, R} \approx-\frac{2}{15} \ln \epsilon-0.25725
$$

and torques

$$
T_{0, S} \approx 0.471996, \quad T_{0, T} \approx \frac{1}{10} \ln \frac{2}{\epsilon}-0.26227, \quad T_{0, R} \approx \frac{2}{5} \ln \epsilon-0.37085 .
$$

In these approximations, the error is asymptotically smaller than some power of $\epsilon$. The requirement (3.7) of force- and torque-free particle provide the linear and angular velocities

$$
\mathscr{U}(\epsilon)=\frac{F_{0, R} T_{0, S}-F_{0, S} T_{0, R}}{T_{0, R} F_{0, T}-T_{0, T} F_{0, R}}, \quad \Omega(\epsilon)=\frac{T_{0, T} F_{0, S}-T_{0, S} F_{0, T}}{T_{0, R} F_{0, T}-T_{0, T} F_{0, R}},
$$

associated with the driving flow.

\subsection{Electro-viscous fields}

Consider next the induced fields in the gap. In view of (2.8), the potential scales as the pressure, suggesting the rescaling

$$
\bar{\varphi}_{0}=\epsilon^{-3 / 2} \Phi_{0}(Z, R ; \epsilon),
$$

where $\Phi_{0}$ is at leading order generated by the translation and rotation subfields of the driving flow. In terms of (4.21) and the inner variables (4.3), Laplace's equation (3.18) adopts the form

$$
\frac{\partial^{2} \Phi_{0}}{\partial Z^{2}}+\epsilon\left(\frac{\partial^{2} \Phi_{0}}{\partial R^{2}}+\frac{1}{R} \frac{\partial \Phi_{0}}{\partial R}-\frac{\Phi_{0}}{R^{2}}\right)=0
$$

At $Z=0$, where $\hat{\mathbf{n}}=\hat{\mathbf{e}}_{z}$, condition (2.8) reads

$$
\frac{\partial \Phi_{0}}{\partial Z}=\frac{\alpha \zeta}{\lambda}\left(\mathscr{U} \frac{\partial P_{0, T}}{\partial Z}+\Omega \frac{\partial P_{0, R}}{\partial Z}\right) ;
$$

At $Z=H$, the surface equations (4.4)-(4.5) yield

$$
\hat{\mathbf{n}} \sim-\hat{\mathbf{e}}_{z}+\epsilon^{1 / 2} \hat{\mathbf{e}}_{r} R+\cdots,
$$

whereby condition $(2.8)$ reads

$$
\begin{aligned}
& \frac{\partial \Phi_{0}}{\partial Z}-\epsilon R \frac{\partial \Phi_{0}}{\partial R}+O\left(\epsilon^{2}\right) \\
& =\frac{\alpha \zeta}{\lambda}\left\{\mathscr{U}\left(\frac{\partial P_{0, T}}{\partial Z}-\epsilon R \frac{\partial P_{0, T}}{\partial R}\right)+\Omega\left(\frac{\partial P_{0, R}}{\partial Z}-\epsilon R \frac{\partial P_{0, R}}{\partial R}\right)\right\}+O\left(\epsilon^{2}\right) .
\end{aligned}
$$


Applying the generic expansion (4.10) to $\Phi_{0}$,

$$
\Phi_{0} \sim \Phi_{0}^{(0)}(Z, R)+\epsilon \Phi_{0}^{(1)}(Z, R)+\cdots,
$$

we find from the leading-order balance of (4.22)

$$
\frac{\partial^{2} \Phi_{0}^{(0)}}{\partial Z^{2}}=0
$$

implying that $\partial \Phi_{0}^{(0)} / \partial Z$ is a function of $R$. Moreover, since the leading-order pressure associated with the translation and rotation subfields is independent of $Z$ (see (4.11) and (4.17)), conditions (4.23) and (4.25) read at leading order

$$
\frac{\partial \Phi_{0}^{(0)}}{\partial Z}=0 \quad Z=0, H_{0}
$$

We conclude that

$$
\Phi_{0}^{(0)}=\Phi_{0}^{(0)}(R)
$$

The dependence of $\Phi_{0}^{(0)}$ upon $R$ will be addressed later on.

Consider now the induced flow within the gap. The slip condition (2.9) in conjunction with the electric-field scaling (4.21) implies $O\left(\epsilon^{-2}\right)$ velocities parallel to wall. The continuity equation (3.20a) then suggests an $O\left(\epsilon^{-3 / 2}\right)$ axial velocity,

$$
\bar{u}_{1}=\epsilon^{-2} U_{1}(Z, R ; \epsilon), \quad \bar{v}_{1}=\epsilon^{-2} V_{1}(Z, R ; \epsilon), \quad \bar{w}_{1}=\epsilon^{-3 / 2} W_{1}(Z, R ; \epsilon) ;
$$

use of the tangential momentum balance results in $O\left(\epsilon^{-7 / 2}\right)$ pressure variations,

$$
\bar{p}_{1}=\epsilon^{-7 / 2} P_{1}(Z, R ; \epsilon) .
$$

Each of fields $U_{1}, V_{1}, W_{1}$ and $P_{1}$ possess the generic expansion (4.10).

Substitution into the continuity and Stokes equations (3.20) respectively yield

$$
\frac{\partial U_{1}}{\partial R}+\frac{U_{1}+V_{1}}{R}+\frac{\partial W_{1}}{\partial Z}=0
$$

and

$$
\frac{\partial P_{1}}{\partial R}=\frac{\partial^{2} U_{1}}{\partial Z^{2}}+O(\epsilon), \quad-\frac{P_{1}}{R}=\frac{\partial^{2} V_{1}}{\partial Z^{2}}+O(\epsilon), \quad \frac{\partial P_{1}}{\partial Z}=O(\epsilon) .
$$

The slip condition (2.9) at the wall, $Z=0$, is

$$
U_{1}=\lambda \zeta \frac{\partial \Phi_{0}}{\partial R}, \quad V_{1}=-\lambda \zeta \frac{\Phi_{0}}{R}, \quad W_{1}=0
$$

from (4.24) and (4.28), the corresponding conditions on the particle, $Z=H$, read

$$
U_{1}=\lambda \zeta \frac{\partial \Phi_{0}}{\partial R}+O(\epsilon), \quad V_{1}=-\lambda \zeta \frac{\Phi_{0}}{R}+O(\epsilon), \quad W_{1}=\lambda \zeta R \frac{\partial \Phi_{0}}{\partial R}+O(\epsilon) .
$$

\subsection{Electro-viscous forces}

Consider now the electro-viscous forces resulting from the gap-scale fields. The lift (3.13) depends only upon the Maxwell stress. Substitution into (2.19) of (3.17), (4.21) and (4.26), in conjunction with (4.29), yields the inner approximation

$$
\begin{aligned}
\mathrm{M}_{1}=-\frac{1}{2} \mid \lambda \epsilon^{-4}\left[\left(\frac{d \Phi_{0}^{(0)}}{d R}\right)^{2} \cos ^{2} \theta\right. & \left.+\left(\frac{\Phi_{0}^{(0)}}{R}\right)^{2} \sin ^{2} \theta\right] \\
& + \text { terms } O\left(\epsilon^{-4}\right) \text { that vanish upon multiplication by } \hat{\mathbf{e}}_{z}+\text { terms } o\left(\epsilon^{-4}\right) .
\end{aligned}
$$


In the inner gap region, (4.24) yields at leading order $\hat{\mathbf{n}} \sim-\hat{\mathbf{e}}_{z}$; subsitition into (3.13) and integration over the entire inner region $(0<R<\infty, 0<\theta,<2 \pi)$ provides the quadrature

$$
6 L_{1}=\frac{\lambda}{2} \epsilon^{-3} \int_{0}^{\infty}\left[\left(\frac{d \Phi_{0}^{(0)}}{d R}\right)^{2}+\left(\frac{\Phi_{0}^{(0)}}{R}\right)^{2}\right] R d R
$$

The calculation of the excess drag and torque requires the induced Newtonian stress $\mathrm{N}_{1}$. Substitution of (3.19) and (4.30) into (2.20) yields the inner approximation

$$
\begin{aligned}
\mathrm{N}_{1}=-\epsilon^{-7 / 2} \mid P_{1}^{(0)} \cos \theta+ & \epsilon^{-3}\left(\hat{\mathbf{e}}_{z} \hat{\mathbf{e}}_{r} \frac{\partial U_{1}^{(0)}}{\partial Z} \cos \theta+\hat{\mathbf{e}}_{z} \hat{\mathbf{e}}_{\theta} \frac{\partial V_{1}^{(0)}}{\partial Z} \sin \theta\right) \\
& +\operatorname{terms} O\left(\epsilon^{-3}\right) \text { that vanish upon multiplication by } \hat{\mathbf{e}}_{z}+\operatorname{terms} o\left(\epsilon^{-3}\right) .
\end{aligned}
$$

Here, two asymptotic terms are explicitly retained, since the contribution of the leading $O\left(\epsilon^{-7 / 2}\right)$ pressure to the excess drag vanishes. Use of (4.24) yields the traction

$\hat{\mathbf{n}} \cdot \mathrm{N}_{1}=\epsilon^{-7 / 2} \hat{\mathbf{e}}_{z} P_{1}^{(0)} \cos \theta$

$$
-\epsilon^{-3}\left(\hat{\mathbf{e}}_{r} R P_{1}^{(0)} \cos \theta+\hat{\mathbf{e}}_{r} \frac{\partial U_{1}^{(0)}}{\partial Z} \cos \theta+\hat{\mathbf{e}}_{\theta} \frac{\partial V_{1}^{(0)}}{\partial Z} \sin \theta\right)+o\left(\epsilon^{-3}\right)
$$

substitution into (3.14) and integration over the entire inner domain yields here

$$
6 D_{1}=\epsilon^{-2} \int_{0}^{\infty}\left(R P_{1}^{(0)}+\frac{\partial U_{1}^{(0)}}{\partial Z}-\frac{\partial V_{1}^{(0)}}{\partial Z}\right)_{Z=H_{0}} R d R
$$

Lastly, the torque (3.16) is also calculated using (4.24) and the two-term approximation (4.35), resulting in the quadrature

$$
8 T_{1}=\epsilon^{-2} \int_{0}^{\infty}\left(\frac{\partial U_{1}^{(0)}}{\partial Z}-\frac{\partial V_{1}^{(0)}}{\partial Z}\right)_{Z=H_{0}} R d R
$$

where again the $O\left(\epsilon^{-7 / 2}\right)$ pressure term in (4.34) does not contribute. (Evidently, the torque about the particle center cannot be affected by the pressure distribution.)

\section{Identical zeta potentials}

The preceding general analysis is valid for uniform surface-charge distributions on the wall and particle, which in the macroscale description are represented by uniform zeta potentials. In this section we consider the detailed calculation of the electro-viscous forces for the relatively simple case of identical particleand wall- zeta potentials. In this case, there is no need to calculate the functional dependence of the leading-order potential (4.29) upon $R$ : it is simply provided by (2.21) with the pressure consisting of the contributions of the translation and rotation subfields

$$
\Phi_{0}^{(0)}=\frac{\alpha \zeta}{\lambda}(\mathscr{U}+\Omega) P^{*}(R) .
$$

With the potential known, it remains to calculate the induced flow. 


\subsection{Induced flow}

In view of $(2.9)$ and (5.1) it is natural to define

$$
\begin{gathered}
U_{1}^{(0)}=(\mathscr{U}+\Omega) \alpha \zeta^{2} \tilde{U}, \quad V_{1}^{(0)}=(\mathscr{U}+\Omega) \alpha \zeta^{2} \tilde{V}, \quad W_{1}^{(0)}=(\mathscr{U}+\Omega) \alpha \zeta^{2} \tilde{W}, \\
P_{1}^{(0)}=(\mathscr{U}+\Omega) \alpha \zeta^{2} \tilde{P} .
\end{gathered}
$$

The differntial equations governing $\tilde{U}, \tilde{V}, \tilde{W}$ and $\tilde{P}$ are obtained from the leading-order balances of (4.31),

$$
\begin{gathered}
\frac{\partial \tilde{U}}{\partial R}+\frac{\tilde{U}+\tilde{V}}{R}+\frac{\partial \tilde{W}}{\partial Z}=0, \\
\frac{\partial \tilde{P}}{\partial R}=\frac{\partial^{2} \tilde{U}}{\partial Z^{2}}, \quad-\frac{\tilde{P}}{R}=\frac{\partial^{2} \tilde{V}}{\partial Z^{2}}, \quad \frac{\partial \tilde{P}}{\partial Z}=0,
\end{gathered}
$$

while the boundary conditions are extracted from (4.32),

$$
\begin{gathered}
\tilde{U}=\frac{d P^{*}}{d R}, \quad \tilde{V}=-\frac{P^{*}}{R}, \quad \tilde{W}=0 \quad \text { at } \quad Z=0, \\
\tilde{U}=\frac{d P^{*}}{d R}, \quad \tilde{V}=-\frac{P^{*}}{R}, \quad \tilde{W}=R \frac{d P^{*}}{d R} \quad \text { at } \quad Z=H_{0} .
\end{gathered}
$$

From (5.3b) $\tilde{P}$ is independent of $Z$. Integration of the the momentum balances and application of the boundary conditions (5.4) yield the velocity profiles

$$
\tilde{U}=\frac{1}{2} \frac{d \tilde{P}}{d R}\left(Z^{2}-H_{0} Z\right)+\frac{d P^{*}}{d R}, \quad \tilde{V}=-\frac{1}{2} \frac{\tilde{P}}{R}\left(Z^{2}-H_{0} Z\right)-\frac{P^{*}}{R} .
$$

Evaluation of $\tilde{W}$ from the continuity equation (5.3a) and the boundary conditions (5.4) eventually yields the differential equation

$$
R^{2} \frac{d^{2} \tilde{P}}{d R^{2}}+\left(R+\frac{3 R^{3}}{H_{0}}\right) \frac{d \tilde{P}}{d R}-\tilde{P}=\frac{12}{H_{0}^{2}}\left[R^{2} \frac{d^{2} P^{*}}{d R^{2}}+\left(R+\frac{R^{3}}{H_{0}}\right) \frac{d P^{*}}{d R}-P^{*}\right]
$$

which, upon substituting (4.12), becomes

$$
R^{2} \frac{d^{2} \tilde{P}}{d R^{2}}+\left(R+\frac{3 R^{3}}{H_{0}}\right) \frac{d \tilde{P}}{d R}-\tilde{P}=\frac{12\left(3 R^{5}-42 R^{3}\right)}{5 H_{0}^{6}} .
$$

While no boundary condition are specified the solution must be finite at $R=0$ and vanish as $R \rightarrow \infty$ (since in the outer region the pressure is clearly $O(1)$ ). For small $R$ a fundamental set of the associated homogenous equation behave like $R$ and $R^{-1}$, respectively, and the particular solution of (5.7) is asymptotic to $-63 R^{3} / 5$. Since the divergent solution must be rejected, $\tilde{P} \sim M R-63 R^{3} / 5$ there. At large $R$ a pair of linearly independent solutions of the associated homogenous equation and a particular solution of (5.7) behave like $R^{-2 \pm \sqrt{10}}$ and $384 / 5 R^{7}$, respectively.

Following O'Neill \& Stewartson [15] and Bike \& Prieve [5], (5.7) has been numerically integrated twice: In the first integration, the initial conditions were obtained assuming $\tilde{P} \sim-63 / 5 R^{3}$ at small $R$; at the second integration, performed with the right-hand side of (5.7) set to zero, the initial behavior $\tilde{P} \sim R$ was assumed. The value of $M$ is selected so that the linear combination of the two solutions decays at infinity. This is equivalent to rejecting the divergent $\sim R^{-2+\sqrt{10}}$ solution there. 


\subsection{Electro-viscous forces}

\subsubsection{Lift}

In view of (4.33) and (5.1) it is natural to define

$$
L_{1}=\frac{\alpha^{2} \zeta^{2}}{\lambda}(\mathscr{U}+\Omega)^{2} \epsilon^{-3} \tilde{L} .
$$

Then,

$$
6 \tilde{L}=\frac{1}{2} \int_{0}^{\infty}\left[\left(\frac{d P^{*}}{d R}\right)^{2}+\left(\frac{P^{*}}{R}\right)^{2}\right] R d R .
$$

Substituting (4.12) and integrating yields

$$
\tilde{L}=\frac{8}{125}
$$

\subsubsection{Excess drag}

In view of (4.30) and (5.2) we define

$$
D_{1}=\alpha \zeta^{2}(\mathscr{U}+\Omega) \epsilon^{-2} \tilde{D}
$$

whereby (4.36) becomes

$$
6 \tilde{D}=\int_{0}^{\infty}\left(R \tilde{P}+\frac{\partial \tilde{U}}{\partial Z}-\frac{\partial \tilde{V}}{\partial Z}\right)_{Z=H_{0}} R d R .
$$

Upon substitution of (5.5), the integrand becomes

$$
\frac{1}{2}\left\{\frac{d}{d R}\left(R H_{0} \tilde{P}\right)+R^{2} \tilde{P}\right\}
$$

since $\tilde{P}$ is zero at $R=0$ and decays faster than $R^{-3}$ at large $R$ we obrain

$$
6 \tilde{D}=\frac{1}{2} \int_{0}^{\infty} R^{2} \tilde{P} d R .
$$

Numerical quadrature readily yields

$$
\tilde{D} \doteqdot 0.2399
$$

As expected, the streaming-potential mechanism results in drag enhancement.

\subsubsection{Torque}

Similarly to (5.11) we define

$$
T_{1}=(\mathscr{U}+\Omega) \alpha \zeta^{2} \epsilon^{-2} \tilde{T} .
$$

Use of (4.37) and (5.2) yields

$$
8 \tilde{T}=\int_{0}^{\infty}\left(\frac{\partial \tilde{U}}{\partial Z}-\frac{\partial \tilde{V}}{\partial Z}\right)_{Z=H_{0}} R d R
$$

Substituting (5.5) we obtain

$$
8 \tilde{T}=\frac{1}{2} \int_{0}^{\infty} H_{0} \frac{d}{d R}(R \tilde{P}) d R
$$

which we integrate by parts to yield

$$
8 \tilde{T}=-\frac{1}{2} \int_{0}^{\infty} R^{2} \tilde{P} d R .
$$

This is the same integral as (5.13), thus

$$
\tilde{T} \doteqdot-0.1799 \text {. }
$$




\section{Unequal zeta potentials}

In the more general case where the particle $\left(\zeta_{P}\right)$ and wall $\left.\zeta_{W}\right)$ zeta potentials differ $\Phi_{0}^{(0)}$ is not directly proportional to the leading-order pressure of the driving flow, and needs therefore to be explicitly calculated. In view of the complications involved, we focus here upon the electro-viscous lift, which is more amenable to experimental observation than the drag and torque enhancements (see $\S 7$ ).

Since the induced potential $\Phi_{0}$ is governed by the linear equation (4.22) together with the boundary conditions (4.23) and (4.25) which, in turn, are linear in the zeta potential, it is conveniently represented as a superposition of two parts: the first corresponding to identical zeta potential $\zeta_{P}$ on both surfaces, and the second with zero particle zeta potential and wall zeta potential $\zeta_{W}-\zeta_{P}$.

As the former problem has already been solved, we focus upon the latter. Condition (4.25) implies that $\Phi_{0}^{(0)}$ is linear in $\zeta_{W}-\zeta_{P}$ as well as the driving-flow pressure associated with translation and rotation. This suggests the representation

$$
\Phi_{0}=\frac{\alpha}{\lambda}\left(\zeta_{W}-\zeta_{P}\right)\left(\mathscr{U} \Phi_{T}+\Omega \Phi_{R}\right)
$$

Both $\Phi_{T}$ and $\Phi_{R}$ possess the generic expansion (4.10). We have established above that the leading term in each expansion is a function of $R$ alone. Its actual calculation requires the analysis of the next asymptotic orders. Consider first $\Phi_{T}$. Here, Laplace's equation (4.22) reads

$$
\frac{\partial^{2} \Phi_{T}^{(1)}}{\partial Z^{2}}=-\frac{\partial^{2} \Phi_{T}^{(0)}}{\partial R^{2}}-\frac{1}{R} \frac{\partial \Phi_{T}^{(0)}}{\partial R}+\frac{\Phi_{T}^{(0)}}{R^{2}}
$$

condition (4.23) yields at $Z=0$

$$
\frac{\partial \Phi_{T}^{(1)}}{\partial Z}=\frac{\partial P_{0, T}^{(1)}}{\partial Z} \quad \text { at } \quad Z=0
$$

and condition (4.25), with zero zeta potential on the particle, reads

$$
\frac{\partial \Phi_{T}^{(1)}}{\partial Z}=R \frac{d \Phi_{T}^{(0)}}{d R} \quad \text { at } \quad Z=H_{0}
$$

Note, using (4.7b), that

$$
\frac{\partial P_{0, T}^{(1)}}{\partial Z}=\frac{\partial^{2} W_{0, T}^{(0)}}{\partial Z^{2}} .
$$

Integration of (6.2) and application of conditions (6.3)-(6.4) in conjunction with (6.5) yields an ordinary differential equation governing $\Phi_{T}^{(0)}$,

$$
R^{2} \frac{d^{2} \Phi_{T}^{(0)}}{d R^{2}}+\left(R+\frac{R^{3}}{H_{0}}\right) \frac{d \Phi_{T}^{(0)}}{d R}-\Phi_{T}^{(0)}=\left.\frac{R^{2}}{H_{0}} \frac{\partial^{2} W_{0, T}^{(0)}}{\partial Z^{2}}\right|_{Z=0} .
$$

Similarly,

$$
R^{2} \frac{d^{2} \Phi_{R}^{(0)}}{d R^{2}}+\left(R+\frac{R^{3}}{H_{0}}\right) \frac{d \Phi_{R}^{(0)}}{d R}-\Phi_{R}^{(0)}=\left.\frac{R^{2}}{H_{0}} \frac{\partial^{2} W_{0, R}^{(0)}}{\partial Z^{2}}\right|_{Z=0}
$$

Substitution of (4.13) and (4.18) yields

$$
\left.\frac{\partial^{2} W_{0, T}^{(0)}}{\partial Z^{2}}\right|_{Z=0}=\frac{4 R^{3}-16 R}{5 H_{0}^{3}},\left.\quad \frac{\partial^{2} W_{0, R}^{(0)}}{\partial Z^{2}}\right|_{Z=0}=-\frac{26 R+R^{3}}{5 H_{0}^{3}},
$$

respectively. The solutions of (6.6)-(6.7) need to remain finite at $R=0$ and decay for $R \rightarrow \infty$. A fundamental set of solutions of the homogenous equation associated with (6.6)-(6.7) behave like $R^{ \pm 1}$ as 
$R \rightarrow 0$ and like $R^{-1 \pm \sqrt{2}}$ as $R \rightarrow \infty$. Particular integrals of (6.6)-(6.7) with the forcing terms as in (6.8) behave for $R \rightarrow 0$ like $-2 R^{3} / 5$ and $-13 R^{3} / 20$, respectively, and like $-32 / 5 R^{3}$ and $-8 / 5 R^{3}$, respectively, as $R \rightarrow \infty$. Similarly to (5.7) the required solutions are obtained through numerical integrations of (6.6)(6.7) and their associated homogenous equations with initial conditions corresponding to the appropriate behavior at $R \rightarrow 0$. The resulting solutions are then superposed so as to ensure the decay at infinity.

With the functions $\Phi_{T}^{(0)}$ and $\Phi_{R}^{(0)}$ evaluated, we now consider the combined electric potential, provided by (5.1) (with $\left.\zeta=\zeta_{P}\right)$ and $(6.1)$

$$
\Phi_{0}^{(0)}=\frac{\alpha \zeta_{P}}{\lambda}(\mathscr{U}+\Omega) P^{*}(R)+\frac{\alpha}{\lambda}\left(\zeta_{W}-\zeta_{P}\right)\left(\mathscr{U} \Phi_{T}^{(0)}+\Omega \Phi_{R}^{(0)}\right)
$$

Substituting into the lift expression (4.33) yields the generalization of (5.8) and (5.10)

$$
\begin{aligned}
L_{1}=\frac{\alpha^{2}}{12 \lambda \epsilon^{3}}\left[\frac{96}{125}(\mathscr{U}+\Omega)^{2} \zeta_{P}^{2}+\left(\zeta_{W}-\zeta_{P}\right)^{2}\left(k_{1} \mathscr{U}^{2}+k_{2} \Omega^{2}\right.\right. & \left.+2 k_{3} \mathscr{U} \Omega\right) \\
& \left.+2 \zeta_{P}\left(\zeta_{W}-\zeta_{P}\right)(\mathscr{U}+\Omega)\left(k_{4} \mathscr{U}+k_{5} \Omega\right)\right]
\end{aligned}
$$

where

$$
\begin{gathered}
k_{1}=\int_{0}^{\infty}\left\{\left(\frac{d \Phi_{T}^{(0)}}{d R}\right)^{2}+\left(\frac{\Phi_{T}^{(0)}}{R}\right)^{2}\right\} R d R, \quad k_{2}=\int_{0}^{\infty}\left\{\left(\frac{d \Phi_{R}^{(0)}}{d R}\right)^{2}+\left(\frac{\Phi_{R}^{(0)}}{R}\right)^{2}\right\} R d R, \\
k_{3}=\int_{0}^{\infty}\left\{\frac{d \Phi_{T}^{(0)}}{d R} \frac{d \Phi_{R}^{(0)}}{d R}+\frac{\Phi_{T}^{(0)} \Phi_{R}^{(0)}}{R^{2}}\right\} R d R, \quad k_{4}=\int_{0}^{\infty}\left\{\frac{P^{*} \Phi_{T}^{(0)}}{R^{2}}+\frac{d P^{*}}{d R} \frac{d \Phi_{T}^{(0)}}{d R}\right\} R d R, \\
k_{5}=\int_{0}^{\infty}\left\{\frac{P^{*} \Phi_{R}^{(0)}}{R^{2}}+\frac{d P^{*}}{d R} \frac{d \Phi_{R}^{(0)}}{d R}\right\} R d R .
\end{gathered}
$$

Performing the quadratures yield

$$
k_{1} \doteqdot 0.0595, \quad k_{2} \doteqdot 0.4214, \quad k_{3} \doteqdot 0.1435, \quad k_{4} \doteqdot 0.2030, \quad k_{5} \doteqdot 0.5649
$$

which completes the lift calculation. Upon setting $\Omega$ to zero, we recover the results of Bike \& Prieve [5], see $\S 7$

\section{Discussion}

In this contribution we employ the generic thin-Debye-layer YSF paradigm to the problem of shearinduced lift. With this paradigm, leading-order $O\left(\delta^{2}\right)$ electro-viscous forces are contributed by both Maxwell and Newtonian stresses. In addition to electrokinetic lift, these stresses also yield excess drag and torque. The calculation of these electro-viscous loads was the subject of the present contribution. For near-contact configurations, the lift scales as $\epsilon^{-3}$, while the drag and torque corrections scale as $\epsilon^{-2}$.

Since we consider at the outset a freely suspended particle, a self-consistent formulation should apparently allow for $O\left(\delta^{2}\right)$ corrections to the particle velocities, e.g. through the boundary condition (2.9). These corrections would be found by imposing the of force- and torque-free conditions, similarly to the above analysis of the driving-flow problem. Rather than pursuing this higher-order mobility problem we have focused upon the more intuitive calculation of the electro-viscous forces. This approach can be generalized to other situations, involving gravity effects. Indeed, in typical experiments the electrokinetic lift is measured indirectly through observations of the equilibrium particle position in the presence of gravity field normal to the wall [4]. 
It is nevertheless worthwhile to estimate the induced particle velocities for a full mobility problem. The induced electro-viscous loads are proportional to either $\epsilon^{-3}$ (lift) or $\epsilon^{-2}$ (excess drag and torque). Symmetry considerations readily show that the lift affects only the velocity normal to the wall, while the excess drag and torque affect the velocity parallel to the wall (as well as the angular velocity, which is of less interest). As is evident from the resistance coefficients (4.19), the effects of excess drag and torque upon lateral motion are comparable; incorporating logarithmic terms at $O(1)$, both excess loads lead to an $O\left(\delta^{2} / \epsilon^{2}\right)$ correction in the velocity parallel to the wall. Moreover, since the resistance to particle motion normal to the wall is $O\left(\epsilon^{-1}\right)$ [21], electrokinetic lift results in a normal velocity of the same $(\delta / \epsilon)^{2}$ scaling. This identical velocity scaling demonstrates the comparable role of all the electro-viscous loads.

That being said, we still consider the prediction of electrokinetic lift to be of greater interest for the experimentalists. This has to do with absence of particle velocity normal to the wall in the absence of electro-viscous effects. Clearly, it is much easier to measure a quantity than a perturbation to one. Moreover, as we already mentioned, a common experimental practice is to measure the lift indirectly through the action of gravity. The correction to the lateral motion is thus of less interest, and the angular velocity is probably of no practical interest.

An important feature of the present analysis has to do with the problem formulation where, following Bike \& Prieve [7], we allow for both particle translation and rotation. This particle motion is set by the imposed shear, with the respective velocities being determined from the conditions of force- and torquefree particle. In the near-contact limit, we actually find that the flow is determined at leading order by the particle rigid-body motion, rather than the animating shear. The contributions of translation and rotation are comparable; for equal zeta potentials, the electro-viscous forces are functions of their sum. Thus, the present results are strongly related to the near-contact analysis of Bike \& Prieve [5], carried out for the case of particle translation. Indeed, artificially setting $\Omega=0$ in the lift expression reproduces the expression of Bike \& Prieve [5].

Since the generic scheme employs an asymptotic expansion in $\delta$, it may appear that the predicted singular terms may impose certain limitations on the validity of the present results. Note however that the velocities induced by the electro-viscous loads all scale as $\epsilon^{-2}$. Thus, the original condition $\delta \ll 1$ is modified to $\delta^{2} \epsilon^{-2} \ll 1$. However, the latter is automatically satisfied by the geometric requirement

$$
\delta \ll \epsilon
$$

which underlies the use of the thin-double-layer macroscale paradigm for near-contact configurations.

The lift-force scaling with $\delta^{2} \epsilon^{-3}$ is the same as in [5]. Since the lift calculated by Bike \& Prieve [5] underestimates experimental data by orders of magnitudes, the same holds for the present results. In the experiments of Alexander \& Prieve [3], with shear rates of about $10 \mathrm{~s}^{-1}$, the Péclet number is about 10. In these experiments, however, $\delta$ is about $10^{-3}$. Clearly, the underlying YSF scaling $\mathrm{Pe}=O\left(\delta^{-2}\right)$ is inappropriate for modeling these experiments. This provides a rational explanation for the experimenttheory discrepancy. The results of the present model would therefore apply only for different experimental settings, with lower salt concentration and higher shear rates. Alternatively, a theoretical understanding of existing experiments requires a different calculation, carried out for $O(1)$ Péclet numbers. In view of the scaling results of YSF, however, understanding streaming-potential phenomena at $\mathrm{Pe}=O(1)$ with $\delta \ll 1$ necessitates a new asymptotic analysis allowing for $O\left(\delta^{-2}\right)$ Hartmann numbers. Such asymptotic model has recently been derived [22]. The application of this model to the problem of shear-induced lift is currently in progress.

Acknowledgements. This research was supported by the Israel Science Foundation (grant no. 114/09).

\section{References}

[1] D. J. Jeffrey. Some basic principles in interaction calculations. In E. M. Torry, editor, Sedimentation of small particles in a viscous fluid, chapter 4, pages 97-124. Computational Mechanics, 1996. 
[2] L. G. Leal. Advanced Transport Phenomena: Fluid Mechanics and Convective Transport Processes. Cambridge University Press, New York, 2007.

[3] B. M. Alexander, D. C. Prieve. A hydrodynamic technique for measurement of colloidal forces. Langmuir, 3 (1987) No. 5, 788-795.

[4] S. G. Bike, L. Lazarro, D. C. Prieve. Electrokinetic lift of a sphere moving in slow shear flow parallel to a wall I. Experiment. J. Colloid Interface Sci., 175 (1995) No. 2, 411-421.

[5] S. G. Bike, D. C. Prieve. Electrohydrodynamic lubrication with thin double layers. J. Colloid Interface Sci., 136 (1990), No. $1,95-112$.

[6] S. G. Bike, D. C. Prieve. Electrohydrodynamics of thin double layers: a model for the streaming potential profile. J. Colloid Interface Sci., 154 (1992), 87-96.

[7] S. G. Bike, D. C. Prieve. Electrokinetic lift of a sphere moving in slow shear flow parallel to a wall II. Theory. J. Colloid Interface Sci., 175 (1995), No. 2, 422-434.

[8] T. G. M. van de Ven, P. Warszynski, S. S. Dukhin. Attractive electroviscous forces. Colloid Surface A, 79 (1993), No. 1, 33-41.

[9] T. G. M. van de Ven, P. Warszynski, S. S. Dukhin. Electrokinetic lift of small particles. J. Colloid Interface Sci., 157 (1993), No. 2, 328-331.

[10] R. G. Cox. Electroviscous forces on a charged particle suspended in a flowing liquid. J. Fluid Mech., 338 (1997), 1-34.

[11] S. M. Tabatabaei, T. G. M. van de Ven, A. D. Rey. Electroviscous sphere-wall interactions. J. Colloid Interface Sci., 301 (2006), No. 1, 291-301.

[12] E. Yariv, O. Schnitzer, I. Frankel. Streaming-potential phenomena in the thin-Debye-layer limit. Part 1. General theory. J. Fluid Mech., 685 (2011), 306-334.

[13] O. Schnitzer, A. Khair, E. Yariv. Irreversible Electrokinetic Repulsion in Zero-Reynolds-Number Sedimentation. Phys. Rev. Lett., 107 (2011), 2783014.

[14] J. B. Keller. Conductivity of a medium containing a dense array of perfectly conducting spheres or cylinders or nonconducting cylinders. J. Appl. Phys., 34 (1963), 991-993.

[15] M. E. O'Neill, K. Stewartson. On the slow motion of a sphere parallel to a nearby plane wall. J. Fluid Mech., 27 (1967), $705-724$.

[16] M. D. A. Cooley, M. E. O'Neill. On the slow rotation of a sphere about a diameter parallel to a nearby plane wall. J. Inst. Math. Applics., 4 (1968), 163-173.

[17] A. J. Goldman, R.G. Cox, H. Brenner. Slow viscous motion of a sphere parallel to a plane wall - I. Motion through a quiescent fluid. Chem. Engng Sci., 22 (1967), 637-651.

[18] M. Van Dyke. Perturbation methods in fluid mechanics. Academic press, New York, 1964.

[19] A. J. Goldman, R.G. Cox, H. Brenner. Slow viscous motion of a sphere parallel to a plane wall - II. Couette flow. Chem. Engng Sci., 22 (1967), No. 4, 653-660.

[20] M. E. O'Neill. A sphere in contact with a plane wall in a slow linear shear flow. Chem. Engrg Sci., 23 (1968), No. 11, $1293-1298$

[21] M. D. A. Cooley, M. E. O'Neill. On the slow motion generated in a viscous fluid by the approach of a sphere to a plane wall or stationary sphere. Mathematika, 16 (1969), 37-49.

[22] O. Schnitzer, I. Frankel, E. Yariv. Streaming-potential phenomena in the thin-Debye-layer limit. Part 2. Moderate Péclet numbers. J. Fluid Mech., (2012), In press. 